\title{
REVISIÓN DOCUMENTAL DE LAS TECNOLOGÍAS DE LA INFORMACIÓN Y LAS COMUNICACIONES (TIC) APLICADAS EN LA EDUCACIÓN SECUNDARIA COLOMBIANA
}
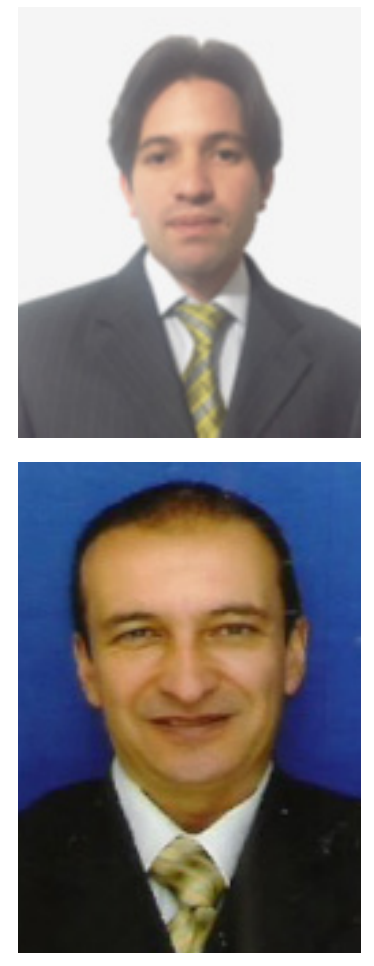

Fecha de recepción: 02/10/2017

\section{Diego Hernández}

Universidad Metropolitana de Educación, Ciencia y Tecnología, UMECIT, Panamá

Institución Educativa Los Comuneros, Colombia

diegohv0003@gmail.com

\section{Benjamín Losada}

Director de Veritas Aid - Red Latino americana de Educación e Investigación Científica, Colombia

veritasaid@gmail.com

\section{RESUMEN}

Con el objeto de evaluar la relevancia de las tecnologías de la información y la comunicación (TIC) en la educación media colombiana, se tomaron antecedentes de investigaciones sobre la materia, adelantados en los departamentos del Cauca y Tolima, así como en los municipios de Soledad Departamento del Atlántico), Muzo y Chiquinquirá (Departamento de Boyacá). En la última población se realizó un estudio de caso alrededor de la Institución Educativa Los Comuneros, mediante la aplicación de entrevistas y encuestas estructuradas a 16 docentes y 53 estudiantes, bajo el criterio de determinar el estado TIC frente al mejoramiento en los procesos de enseñanza en el país suramericano: a través del análisis documental de "La innovación Educativa en Colombia: buenas prácticas para la Innovación y las TIC en Educación” (2016) y de “Tecnología para la transformación y el mejoramiento de la educación: experiencias de éxito y expectativas para el futuro", (2014) se pudo evidenciar el mejoramiento de los estudiantes en las pruebas internas gubernamentales de selección múltiple denominadas SABER 11 cuando se aplican las TIC. Sumado a lo anterior serealizóla revisión documental de los estudios adelantados por la Fundación Santillana y el Ministerio de Educación 
Revisión documental de las tecnologías de la información y las comunicaciones (TIC) aplicadas en la educación secundaria colombiana, Diego Hernández - Benjamín Losada (57:76)

Nacional de Colombia (MEN), permitió reflexionar con la sumatoria de elementos ya relacionados sobre el tema propuesto, generando como resultados y conclusiones que se debe crear un modelo didáctico a partir de las TIC, fortalecer la capacitación docente y adecuar la infraestructura de los establecimientos educativos a las tecnologías en boga, todo en función del mejoramiento de la calidad de la educación.

Palabras clave: Nuevas tecnologías; procesos de enseñanza; calidad educativa; modelo didáctico.

\title{
DOCUMENTARY REVIEW OF INFORMATION TECHNOLOGIES AND COMMUNICATIONS (ICT) APPLIED IN COLOMBIAN SECONDARY EDUCATION
}

\begin{abstract}
In order to evaluate the relevance of information and communication technologies (ICT) in the colombian middle education, it was taken the research history on the subject, advanced in the departments of Cauca and Tolima, as well as in the municipalities of Soledad Department of Atlántico), Muzo and Chiquinquirá (Department of Boyacá). In the last population, a case study was conducted at the Los Comuneros Educational Institution, through the application of structured interviews and surveys in 16 teachers and 53 students, under the criterion of determining the state in which they are located, of the "Educational innovation in Colombia: good practices for innovation and ICT in education" (2016) and "Technology for the transformation and improvement" of education: experiences of success and expectations for the future ", (2014) evidence the improvement of students in public tests of multiple choice called SABER 11 when ICT is applied. In addition, the documentary review of the studies carried out by the Santillana Foundation and the Colombian Ministry of National Education (MEN), reflected on the sum of elements related to the proposed topic, generating as results and conclusions that a didactic model should be created to from ICT, strengthen teacher training and adapt the infrastructure of educational sites to the technologies in vogue, all in terms of improving the quality of education.
\end{abstract}

Key words: New technologies, teaching process, educational quality, didactic model. 
Revisión documental de las tecnologías de la información y las comunicaciones (TIC) aplicadas en la educación secundaria colombiana, Diego Hernández - Benjamín Losada (57:76)

\section{INTRODUCCIÓN}

El surgimiento y continuo desarrollo de las nuevas tecnologías a nivel mundial, trae retos en diferentes campos del conocimiento y esto se ve reflejado principalmente en el ámbito educativo, el cual requiere un profundo análisis que conlleve a determinar cómo mejorar el proceso de enseñanza a partir de las TIC y como crear políticas educativas que contribuyan a mejorar la infraestructura, desarrollar aplicaciones (software, webs y portales) y dotar con dispositivos que apoyen significativamente al mejoramiento continuo de la educación del país.

El Informe Nacional de Competitividad 2016-2017 en el campo de educación media, establece en una de sus recomendaciones que se debe elaborar un modelo para el uso pedagógico de las TIC, considerando que a pesar de los grandes esfuerzos en dotación de computadores, materiales tecnológicos e infraestructura no es suficiente si no se tiene una estrategia educativa integral. Por lo tanto, sugieren que el MEN y las diferentes Secretarias de Educación unan esfuerzos y lideren el diseño de un modelo orientado al uso pedagógico de las TIC en el aula, capacitación docente y gestión tecnológica en las Instituciones Educativas, que garanticen la realización de contenidos pertinentes. (Consejo Privado de Competitividad, 2017).

\section{SITUACIÓN PROBLEMÁTICA DEL CONTEXTO}

Con el crecimiento vertiginoso de las tecnologías y la nueva sociedad del conocimiento es clara la necesidad de asumir desde la educación secundaria retos que supone la integración TIC para mejorar la calidad educativa, crear nuevas competencias y mejorar la formación integral de las personas.

En Colombia se ha trabajado en marcos que buscan lograr este objetivo y muestra de ello fue el Plan Decenal de Educación 2006-2016 que trata de garantizar el acceso, uso y apropiación de las TIC en los ámbitos de enseñanza-aprendizaje; sin embargo los estudios realizados por instituciones como la Organización de las Naciones Unidas para la Educación, la Ciencia y la Cultura (UNESCO) en 2010, demuestran que la educación del país en cuanto a la integración de las TIC, tiene un camino largo por recorrer ya que se requiere mayor infraestructura informática al contarse 12 estudiantes por equipo de cómputo sumada a la baja conectividad calculada en un $75 \%$ para establecimientos de educación primaria, secundaria y media, lo cual sugiere que la mayoría de los estudiantes podría enfrentar, en ocasiones, limitaciones en términos del uso de Internet con propósitos pedagógicos.

La formación docente no escapa de la baja formación en TIC: las estadísticas de acceso que ofrece el Ministerio del ramo a través del programa SoyTIC, concluye que entre 2011 y 2014 se han 
Revisión documental de las tecnologías de la información y las comunicaciones (TIC) aplicadas en la educación secundaria colombiana, Diego Hernández - Benjamín Losada (57:76)

graduado alrededor de 43.940 profesionales en ciencias de la educación cifra muy lejana a los 3.191 docentes que han recibido formación continuada en competencias TIC bajo la oferta gratuita gubernamental. (Cárdenas, 2015).

Sin embargo, es de aclarar que las TIC no necesariamente se aprenden de manera formal, ya que existen docentes que aprenden de manera autodidacta y refuerzan sus conocimientos, pero, faltan registros que midan el autoaprendizaje en el uso, apropiación y aplicación de las mismas por parte de los docentes que imparten su enseñanza en la educación media colombiana.

En el año 2016 caso más reciente, la innovación tecnológica en la última década incorporando las TIC en el aula tanto en educación básica como en media, donde en el estudio realizado por el MEN y publicado en el libro de "La Innovación Educativa en Colombia", arroja datos relevantes con el fin de cumplir con el objetivo en materia de política tecnológica en educación de apoyar el desempeño de los estudiantes en las áreas básicas, su vida cotidiana y que se vea reflejado en las pruebas nacionales e internacionales. Según el estudio los beneficios de utilizar e incorporar las TIC se dan en gran medida por disminuir la deserción escolar, y desarrollar nuevas habilidades. (Bedoya, Hernández, Rivera \& Ferro, 2016).

Bakia, Murphy, Anderson y Trinidad (2011) consideran que, mediante clases en compañía del docente y uso apropiado de las tecnologías, estas impactan en la calidad de la educación y contribuyen a reforzar el proceso de aprendizaje del estudiante generando un mejor desempeño en sus competencias, especialmente cuando se interactúa con videos, sitios webs, y simulaciones, entre otros.

Si volvemos a Bedoya et al. (2016) encontramos que en Colombia entre el 2014 y 2016 se han formado a más de 150.000 docentes y entregado más de 600.000 terminales, lo que implica sustancial mejora: un $80 \%$ de estudiantes matriculados en el país en establecimientos públicos tienen acceso a Internet, y más de 30.601 sedes de instituciones educativas fueron dotas de infraestructura, computadores y conectividad. Por ultimo asegura que las políticas educativas han impulsado la creación de contenidos digitales y la formación docente.

De cara a la educación rural se encuentra que la brecha es muy alta ya que el $40 \%$ de estudiantes de escuelas rurales no leen información en la web, $32 \%$ no usan redes sociales y $25 \%$ no navega por internet. De igual forma los porcentajes en instituciones educativas urbanas son de $26 \%, 13 \%$ y $8 \%$ respectivamente y a nivel privado es de; $18 \%, 6 \%$ y $2 \%$. Incluso a nivel nacional, el $61 \%$ de estudiantes o jóvenes tienen un nivel limitado en el uso de las TIC, generando como resultado en promedio la utilización de redes, webs, elementos o herramientas TIC un día a la semana.

También se evidencia una gran desigualdad en algunos departamentos como Chocó, Sucre, Cauca, Córdoba ya que el $60 \%$ de los jóvenes no utilizan dichos medios para la realización de 
Revisión documental de las tecnologías de la información y las comunicaciones (TIC) aplicadas en la educación secundaria colombiana, Diego Hernández - Benjamín Losada (57:76)

de tareas o en su defecto es muy bajo el uso de estos medios para la obtención de tareas específicas.

\section{Antecedentes Investigativos en TIC en la educación media Colombiana.}

El creciente desarrollo de diferentes medios, herramientas TIC y Webs, conlleva un serio cuestionamiento de como dichas tecnologías favorecen los diferentes campos de acción, principalmente en el entorno educativo, lo cual lleva a indagar como estas repercuten en el proceso de enseñanza y su implementación en la educación media colombiana.

Lo anterior, ha llevado a los investigadores en el área de la educación a adelantar estudios en diferentes regiones, que ayuden a determinar de manera crítica y objetiva el estado de las TIC en la educación media. Por consiguiente, se muestra a continuación una breve referencia de diferentes investigaciones desarrolladas:

En el año 2012, a nivel nacional se desarrolló una investigación en la Institución Educativa Núcleo Escolar Rural del municipio de Corinto (Cauca), donde enfatizan en la apropiación, uso y aplicación de las TIC en los procesos pedagógicos para mejorar el desempeño académico de los estudiantes; resalta que existen docentes que desconocen el potencial de la tecnología y por ende no se usan ni se aplican su ámbito haciendo necesaria la implementación de estas a la educación. (Muñoz, 2012).

Muñoz (2012) concluye que se sembró un proceso de transformación de la actitud de los maestros frente al proceso formativo y hacia la integración de las TIC en su actividad con el apoyo de informática básica en capacitaciones virtuales y presenciales, frente a las exigencias cognitivas, mejorando la disposición para el uso de estas herramientas mediante la creación de blog, Web 2.0 e interacción con otras herramientas tecnológicas.

En el municipio de Soledad (Departamento de Atlántico), se realizó una investigación de tipo exploratorio enfocado a diagnosticar el uso de las TIC en estudiantes de colegios oficiales en los grados 10 y 11. Para ello tomaron como muestra a 312 estudiantes de diferentes instituciones del municipio de Soledad, teniendo como medio de recolección de información entrevistas y encuestas.

En el caso de las entrevistas, el objetivo era medir el potencial que tienen los estudiantes sobre el manejo de las TIC y las oportunidades al usar estos medios. Para las encuestas las preguntas iban orientadas a medir utilidad y beneficios de la enseñanza recibida en el manejo de las herramientas y la disposición hacia el uso de las TIC. (Castellar, 2011). 
Revisión documental de las tecnologías de la información y las comunicaciones (TIC) aplicadas en la educación secundaria colombiana, Diego Hernández - Benjamín Losada (57:76)

La investigación arrojo como conclusión que se requieren cambios profundos en el papel que desempeñan los diferentes actores involucrados en el proceso de enseñanza-aprendizaje del municipio, que conlleve a romper el estancamiento en cultura tecnológica además de explotar el interés de los jóvenes por este mundo, aprovechando sus deseos e intereses, para superar inexperiencia y obstáculos, dando rienda suelta a su potencial latente. Por tanto invita a entes gubernamentales y otro tipo de actores para avanzar a todas las zonas apartadas sin acceso a salas de informática y espacios para el desarrollo adecuado de las TIC en el aula, en función del desarrollo equitativo. (Castellar, 2011).

Hernández y Martínez (2014) en su investigación sobre aplicación de herramientas web 2.0 para simulación de situaciones de optimización matemática en la institución educativa San Marcos del Municipio de Muzo (Departamento de Boyacá), observan que la incorporación de las TIC debe dirigir el proceso de enseñanza-aprendizaje a una nueva concepción educativa; muy pocos docentes de dicha institución han realizado capacitaciones para el manejo y uso de las nuevas tecnologías, aunque cabe resaltar que la mayoría muestra interés para informarse en el tema por otros medios como internet, libros y periódicos.

Se determinó, que el cambio no debe constituir únicamente en cambiar el papel y el lápiz por el ordenador y la impresora sino en la forma en la que se utilizan las nuevas herramientas. Este cambio profundo en la metodología educativa, que no consiste en utilizar las nuevas herramientas TIC (NTIC) con métodos tradicionales, debe afectar a la enseñanza en pro de las necesidades individualidades del alumno, a través de la interactividad, creando un nuevo marco de relaciones, fomentando el trabajo colaborativo, renovar continuamente y hacer seguimiento a los proyectos educativos, para mejorar la enseñanza con criterios de calidad, equidad innovación y pertinencia; propiciando el uso NTIC.

Se hace necesario hacer esfuerzos para derribar obstáculos institucionales y mejorar las políticas públicas implantadas tanto dentro como fuera de los estamentos educativos, que son los que de alguna forma impiden conseguir beneficios significativos a partir de las TIC. Esto ayudaría a reducir las estadísticas de deserción, lograr desempeños más altos en pruebas de conocimiento y aumentar significativamente los niveles de destrezas de pensamiento de los estudiantes. (Castellar, 2011, p. 87).

En el departamento del Tolima, se desarrolló un trabajo investigativo denominado "Estado actual de la aplicación de las TIC en los colegios que imparten el ciclo de educación media de las zonas rurales del municipio de Ibagué", desarrollado en el año 2010, que tomó 4 colegios ubicados en corregimientos o veredas de la cabecera municipal de su ciudad capital, Ibagué, donde a través de una investigación cualitativa se buscaba descubrir la forma en que se estaba dando el uso de las TIC en relación con los procesos de enseñanza-aprendizaje. (Tique, 2010). 
Revisión documental de las tecnologías de la información y las comunicaciones (TIC) aplicadas en la educación secundaria colombiana, Diego Hernández - Benjamín Losada (57:76)

Dentro de los principales hallazgos de esta investigación se encuentra que las instituciones cuentan con sala de informática, sin embargo no disponen de infraestructura para conectividad de Internet, a lo que se suma una mayoría de estudiantes sin capacitación bajo entornos virtuales, y/o cursos a través de internet.

En los colegios, no se realizan prácticas académicas para inducirlos a trabajar y aprender con las TIC derivado de docentes no capacitados en entornos virtuales, sin conocimiento del plan nacional TIC al punto de no reconocer los navegadores más comunes para buscar información.

La anterior investigación recomienda que los docentes que se encargan de llevar a cabo el proceso de enseñanza en las Instituciones Educativas de las zonas rurales, sean capacitados en TIC a través de programas institucionalizados desde los Ministerios de Educación y de las Tecnologías de la Información y de la Comunicación, para qué ésta capacitación sea transferida de manera eficaz hacia los estudiantes de estas Instituciones Educativas. (Tique, 2010, p. 80).

Parra (2011) considera en cuanto a la relación de la escuela y la formación docente que es sorprendente que siga intacto la aproximación a las instituciones, al conocimiento y a la continua capacitación docente en TIC.

En este sentido, tal vez el fracaso de los programas de alfabetización, capacitación, entrenamiento o educación de los maestros es el triunfo silencioso de las prácticas culturales y los bordes. Quizá es inadecuado simplificar la pregunta en términos de fracaso-triunfo, pero lo que sí interesa es hacer eco de lo expuesto en este texto para encontrar razones para explorar otras preguntas y referentes en cuanto a las TIC y la educación. Y es allí donde, precisamente, las aproximaciones históricas pueden dar luces para avivar y, sobre todo, fundamentar y contextualizar nuevas preguntas, críticas y propuesta. (Parra, 2011, p. 156).

En consecuencia, una etapa de revolución educativa con miras a mejorar la enseñanza y aprendizaje, debe considerar cuales son los diversos factores que podrán permitir la añorada calidad educativa integrada con las TIC, en función de las metas propuestas. (Visbal, 2018), para lo cual es decisivo realizar estudios de caso como los ventilados en este trabajo y profundizar en el estado de las herramientas TIC desarrolladas e implementadas en pro de la calidad educativa como hecho que trasciende metas de cobertura.

Ulate (2012) apunta a un planteamiento claro para educadores a cerca de la relevancia TIC, principalmente en la educación primaria y secundaria, dado que estas permiten la transformación de Instituciones públicas y privadas, estudiantes, padres o acudientes y docentes.

Los recursos tecnológicos apoyan y generan la adquisición de aprendizajes mediante ambientes simulados y la navegación cibernética, por lo que en Colombia el MinTIC debe incrementar el acceso de la ciudadanía a dichas tecnologías y dotar de infraestructura las diversas instituciones educativas. (Hermosa 2015). 
Revisión documental de las tecnologías de la información y las comunicaciones (TIC) aplicadas en la educación secundaria colombiana, Diego Hernández - Benjamín Losada (57:76)

\section{Las TIC y las NTIC}

Actualmente, los términos TIC y NTIC se relacionan con las diferentes herramientas tecnológicas que permiten una mejor comunicación con el campo educativo y la forma como se transmite la información, es decir, se acostumbra a llamar la incorporación de las tecnologías en ambos sentidos; sin embargo, es importante precisar estos términos como se muestra en la tabla 1.

Tabla 1.

Definición de TIC y NTIC seguin Castells (1998), Cabero (2000) y Meneses (2007).

\begin{tabular}{ll}
\hline & \multicolumn{1}{c}{ TIC } \\
\hline 1. & Videos. \\
2. Televisión. \\
3. Fotografia. \\
4. Diapositivas. \\
5. Web 2.0 \\
6. Correo electrónico \\
7. Tutoriales \\
8. Audios \\
9. Hojas de calculo
\end{tabular}

Castells (1998), concibe las TIC como la agrupación de las tecnologías que han sido desarrolladas en los diferentes campos de la informática, las telecomunicaciones, televisión, radio y una serie de aplicaciones.
NTIC

Sabemos que el desarrollo tecnológico a nivel mundial crece a gran escala lo cual sugiere que dichas tecnologias evolucionan constantemente, lo cual le da el sentido de "Nuevas" a las TIC, es decir, Nuevas tecnologias de información y comunicación siendo una transición entre lo tradicional y lo actual, en tal sentido entre las nuevas tecnologias podriamos nombrar las webs 3.0 , $4.0,5.0$ (consideradas webs semánticas). Webs 6.0 y 7.0 (webs de redes sensoriales), las simulaciones, la robótica e incluso las app, que han vivido una transformación $y$ evolución constante a través de los años, lo anterior se puede sustentar según la definición a través de los años de varios autores:

Para Cabero (2000), las NTIC se convierten en una serie de instrumentos técnicos que giran en torno a los nuevos descubrimientos en información, es decir, a los nuevos avances de los distintos medios de los cuales se crean nuevas herramientas, almacena, recupera, y transmiten información de manera más rápida utilizando códigos desde la hipermedia, lo cual conlleva a manejar una gran de información.

Por su parte, Meneges (2007), considera el termino de nuevas tecnologias al conjunto de instrumentos tecnológicos como el computador, las redes, la virtualidad, que giran en tomo de las telecomunicaciones, la informática y medios audiovisuales que facilitan a las personas el interactuar con todo tipo de información.

Las NTIC generan potencialidades: nuevos entornos de comunicación, nuevas formas de acceder, generar y transmitir la información. Su incorporación a la educación lleva a la des formalización, la flexibilidad provoca cambios en el sistema educativo, un nuevo rol de profesor y alumno, etc. Una nueva realidad que da lugar a nuevos entomos educativos. (Meneses, 2007, p. 73).

Fuente: Hernández y Lossada (2018) 
Revisión documental de las tecnologías de la información y las comunicaciones (TIC) aplicadas en la educación secundaria colombiana, Diego Hernández - Benjamín Losada (57:76)

En síntesis, las NTIC son todas aquellas tecnologías y medios que nos permiten comunicarnos y acceder a la información de una forma más rápida y precisa, en donde la palabra "nueva" toma sentido en la vertiente de los cambios generados y la creciente innovación tecnológica que generan cada día mayor acceso a la información y a la comunicación, separando de cierta forma las herramientas tradicionales TIC. Las NTIC aplicadas en la educación estaría determinada a mejorar la forma como se concibe la enseñanza - aprendizaje donde se busca que el estudiante se le facilite el aprendizaje por medio de las herramientas tecnológicas existentes y lógicamente, que el docente se convierta en un guía e intérprete de tal proceso.

\section{METODOLOGÍA}

Con el objetivo específico de determinar las herramientas TIC aplicadas en el proceso de enseñanza en la educación media, no solo se escudriñaron investigaciones recientes, sino que se realizó una investigación en la Institución Educativa Los Comuneros del municipio de Chiquinquirá del departamento de Boyacá - Colombia.

La metodología adecuada a esta situación es la Investigación acción ya que plantea una referencia que debe ser acorde a labor docente, dándole importancia al trabajo del profesor, para diseñar un modelo didáctico de las TIC en el proceso de enseñanza en la educación media: solucionar problemas en el aula, que le da sentido a la investigación acción; igualmente, al indagar, mejorar y perfeccionar la práctica docente mediante espacios de reflexión, desarrollando teorías sobre su acción, y abriendo espacios para el desarrollo profesional. (Latorre, 2003).

En esta investigación se utilizó una metodología mixta; cualitativa y cuantitativa. La primera permite descubrir y completar preguntas investigativas basadas en métodos de recolección de datos partiendo de la descripción, la observación y gracias a su flexibilidad moverse entre el evento y la interpretación, entre las respuestas y el desarrollo de la teoría.

El enfoque cuantitativo brinda la posibilidad de utilizar la recolección y análisis de datos para contestar a preguntas generadas y establecidas previamente, con el fin de confiar en la medición numérica, el conteo, la estadística y determinar cómo se aplican las Webs y las herramientas TIC en la Institución Educativa los Comuneros (IEC).

Los sujetos del estudio lo conforman 350 estudiantes distribuidos en 9 sedes. La principal cuenta con 181 estudiantes desde grado 6 a 11: un directivo docente y 8 docentes de aula; a su vez las otras 8 sedes cuentan con 169 estudiantes distribuidos en Carapacho Alto, Carapacho Bajo, Arboledas, Sasa Alto, Sasa, Moyavita La Capilla, Moyavita, Santa Teresa y Balsa Arriba. 
Revisión documental de las tecnologías de la información y las comunicaciones (TIC) aplicadas en la educación secundaria colombiana, Diego Hernández - Benjamín Losada (57:76)

Dado que la población es relativamente pequeña, para mayor exactitud se efectuó la investigación con los 16 docentes pertenecientes a la IEC, y se escogieron la totalidad de estudiantes de grado once y décimo que suman entre ambos grados 53 estudiantes.

En cuanto a la recolección de la Información se realizó en 3 fases, tal como se detalla en la Tabla 1 con base en la metodología mixta que incluye aplicación de encuestas, entrevistas y análisis de los antecedentes investigativos.

Tabla 2.

Fase, objeto de estudio y técnicas e instrumentos de recogida de información empleados en el trabajo de campo.

\begin{tabular}{|c|c|c|}
\hline FASE & OBJETO DE ESTUDIO & $\begin{array}{c}\text { TECNICAS E } \\
\text { INSTRUMENTOS DE } \\
\text { RECOGIDA DE } \\
\text { INFORMACION }\end{array}$ \\
\hline Primera fase & $\begin{array}{l}\text { - Conocimiento de Herramientas } \\
\text { TIC y Webs. } \\
\text { - Razones por las cuales es } \\
\text { importante el uso de las nuevas } \\
\text { tecnologías. } \\
\text { - Medios en los cuales han } \\
\text { recibido formación en TIC y } \\
\text { Webs. } \\
\text { - Herramientas TIC y Webs que } \\
\text { más se implementan en el } \\
\text { desarrollo de la praxis } \\
\text { pedagógica. } \\
\text { Resultados obtenidos al } \\
\text { implementar las TIC en los } \\
\text { procesos de enseñanza- } \\
\text { aprendizaje. }\end{array}$ & $\begin{array}{c}\text { Encuestas a Docentes y } \\
\text { estudiantes. } \\
\text { Formularios }\end{array}$ \\
\hline Segunda fase & $\begin{array}{l}\text { - Causas que impiden el } \\
\text { desarrollo de espacios que } \\
\text { involucren las TIC en el proceso } \\
\text { de enseñanza. } \\
\text { - Herramientas en las cuales les } \\
\text { gustaría innovar para el } \\
\text { desarrollo de la práctica } \\
\text { pedagógica. }\end{array}$ & Entrevista a docentes \\
\hline Tercera fase & $\begin{array}{l}\text { - Estado de las TIC en Colombia, } \\
\text { basado en estudios previos. }\end{array}$ & Revisión Documental \\
\hline
\end{tabular}

Fuente: Hernández y Lossada (2018) 
Revisión documental de las tecnologías de la información y las comunicaciones (TIC) aplicadas en la educación secundaria colombiana, Diego Hernández - Benjamín Losada (57:76)

\section{Encuesta a Docentes:}

Se realizó la encuesta a los 16 docentes que están en ejercicio de su labor en la Institución Educativa, por ser los directamente involucrados en el proceso de formación del estudiantado y que cuentan con una antigüedad entre los 3 y 17 años en el establecimiento, con el objetivo de percibir desde su experiencia como involucran las TIC en el proceso de enseñanza.

Se aplicó un formulario de encuesta, con estudio de validez y fiabilidad, compuesta por: a) un cuadro de referencias del perfil docente. b) 8 preguntas de tipo abierta y cerrada, en aras de encontrar el estado de formación del docente en manejo y uso de las TIC como por ejemplo; manejo de diapositivas, videos, tutoriales, Web 2.0 y NTIC como; apps educativas, simuladores, webs (5.0, $6.0 \mathrm{y} / \mathrm{o} 7.0)$, robótica entre otros que se manejan en la praxis pedagógica.

\section{Encuesta a estudiantes:}

Como parte imprescindible en el campo educativo, se encuestó a 53 estudiantes de grado $10^{\circ}$ y $11^{\circ}$, de los cuales 25 son hombres y 28 mujeres con edades oscilantes entre los 16 y 19 años con el objeto de examinar la visión del proceso de aprendizaje, tomando como partida como sus docentes involucran las TIC y las Webs en el mismo.

De igual forma se utilizó un formulario de encuesta, en el cual se relacionan las mismas preguntas aplicadas a los docentes, pero desde el punto de vista del estudiante, con el fin de contrastar las respuestas suministradas.

\section{Entrevista a docentes:}

En esta segunda fase, la finalidad fue profundizar y complementar las preguntas y respuestas generadas en la primera fase. Por consiguiente, la entrevista busco caracterizar en primer lugar los estudios alcanzados por los docentes, de los cuales se obtuvo que 2 docentes son magísteres y actualmente doctorandos en educación, y los restantes 14 docentes son egresados de diferentes licenciaturas.

Es importante aclarar que el sistema colombiano para el ingreso a la carrera docente a nivel público, contempla 3 posibilidades en el estatuto de profesionalización docente, establecido en el decreto 1278 de 2002, el cual define el concurso para la carrera docente; aborda que solo podrán ser nombrados normalistas, licenciados o profesionales en otros campos que realicen algún curso de pedagogía.

Luego de establecer los estudios alcanzados, se realizaron preguntas estructuradas, con la finalidad de establecer las causas que impiden el desarrollo de espacios que involucren las TIC en el proceso de enseñanza, expectativas en torno a las mismas TIC y caracterización de herramientas que en criterio de cada docente permitan innovar la práctica pedagógica. 
Revisión documental de las tecnologías de la información y las comunicaciones (TIC) aplicadas en la educación secundaria colombiana, Diego Hernández - Benjamín Losada (57:76)

\section{Referentes Documentales}

En esta tercera fase, se buscó complementar la información obtenida en el estudio de caso, consultando referentes documentales más amplios a nivel nacional, con 2 libros "La innovación Educativa en Colombia: buenas prácticas para la Innovación y las TIC en Educación” (2016) y “Tecnología para la transformación y el mejoramiento de la educación: experiencias de éxito y expectativas para el futuro", (2014). En este último, la fundación Santillana junto con la UNESCO realiza un análisis sobre las condiciones e impacto TIC para mejorar la calidad de la educación. El dos primeros reflejan ejemplos de prácticas pedagógicas exitosas que pueden servir de ejemplo para mejorar en el futuro junto con la aplicación de las NTIC, el segundo nos brinda un análisis detallado del impacto directo de las NTIC en los resultados de las pruebas saber aplicadas en el estado colombiano comparadas en relación a los estudiantes con mayor puntaje y la frecuencia de uso de dichas tecnologías. Lo anterior se complementa junto a las investigaciones mencionadas en los antecedentes en diferentes municipios y/o departamentos de Colombia como en Soledad (Atlántico), Corinto (Cauca), Muzo (Boyacá) y en el departamento de Tolima, los cuales estaban encaminados a determinar el uso, manejo e infraestructura de las TIC en los establecimientos los establecimientos educativos del país.

\section{Limitaciones}

1. El desinterés de algunos docentes en capacitarse sobre herramientas tecnológicas, supone un reto para motivarlos y hacerles entender la importancia de incorporar estas herramientas a la educación.

2. La ubicación geográfica de algunos municipios colombianos, con escasa conectividad a Internet en zonas rurales lo que se traduce en demasiado tiempo para cumplir tareas que en otros lugares se realizan fácilmente.

3. La falta de conocimiento en un gran número de aplicaciones tecnológicas que pueden favorecer el desarrollo de las clases y enriquecerlas con didácticas que contribuyan al mejoramiento del razonamiento crítico y práctico del estudiante. 
Revisión documental de las tecnologías de la información y las comunicaciones (TIC) aplicadas en la educación secundaria colombiana, Diego Hernández - Benjamín Losada (57:76)

\section{Resultados}

De acuerdo con la tabulación y el análisis de instrumentos aplicados en el estudio de caso para docentes (Figura 1) se precisa que:

- El 100\% de los docentes encuestados asegura tener algún tipo de conocimiento en el uso de las TIC.

- El 68,75\% de los docentes que usan las TIC en el desarrollo de su praxis pedagógica, aseguran estas facilitan su labor dado que los estudiantes incrementan su creatividad e imaginación; el 37,5\% restante consideran que estas desarrollan habilidades de participación y acciones responsables generando mayor autonomía por parte del estudiante mejorando además sus competencias en la solución de problemas.

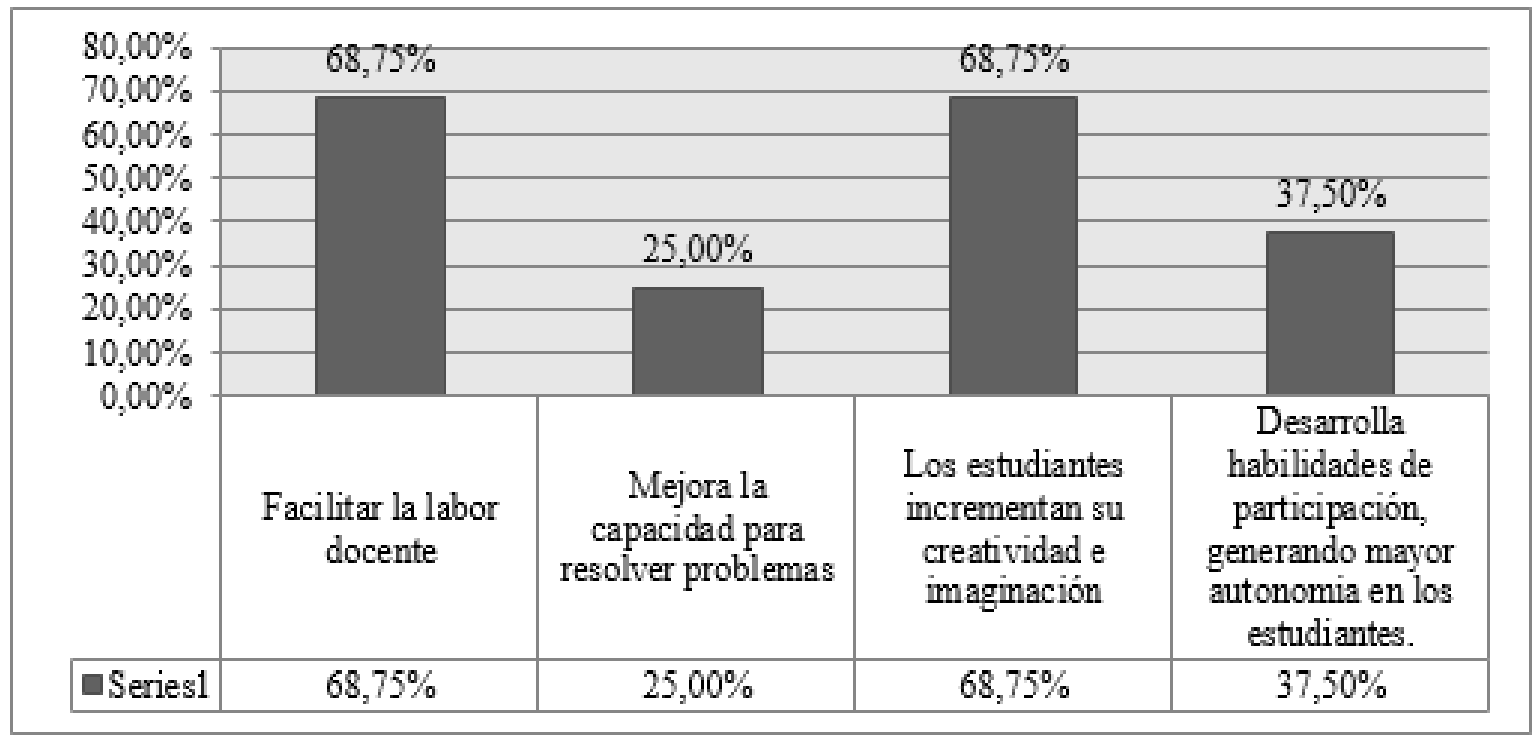

Figura 1 Porcentaje de respuesta a la pregunta: ¿Cuál es el resultado que ha experimentado como docente, al involucrar las TIC en el proceso de enseñanza?

Fuente: Hernández y Lossada (2018)

- El 62,50\% de los docentes aseguran que no han obtenido formación en el manejo de las NTIC, mientras que el 37,50\% de los docentes que han adquirido formación en el manejo y uso de las NTIC, los cursos recibidos hacen parte de los programas desarrollados por políticas educativas del MEN como: EDUTIC que es un programa de capacitación docente ofrecido por el operador Computadores para Educar. TECNOTIC diplomado desarrollado por el Ministerio de las TIC dirigido a docentes de tecnología e informática en el año 2016, este último basado en la creación de apps educativas. 
- En cuanto a las herramientas TIC que utilizan con mayor frecuencia en el desarrollo de las clases, cabe resaltar para este caso; cada herramienta podía llegar al $100 \%$, ya que al aplicar la encuesta se podían seleccionar todas las opciones o algunas de estas, por lo que los docentes muestran su preferencia por videos $68,75 \%$, fotografías $50 \%$, diapositivas $43,75 \%$, correo electrónico $37,50 \%$, video tutoriales $25 \%$, simulaciones $18.75 \%$, foros web y blogs $18.75 \%$ Por su parte el $25 \%$ de los docentes respondieron que han utilizado otros tipos de herramientas para enriquecer el aprendizaje y enseñanza a través de las NTIC como son apps educativas, realidad aumentada y robótica.

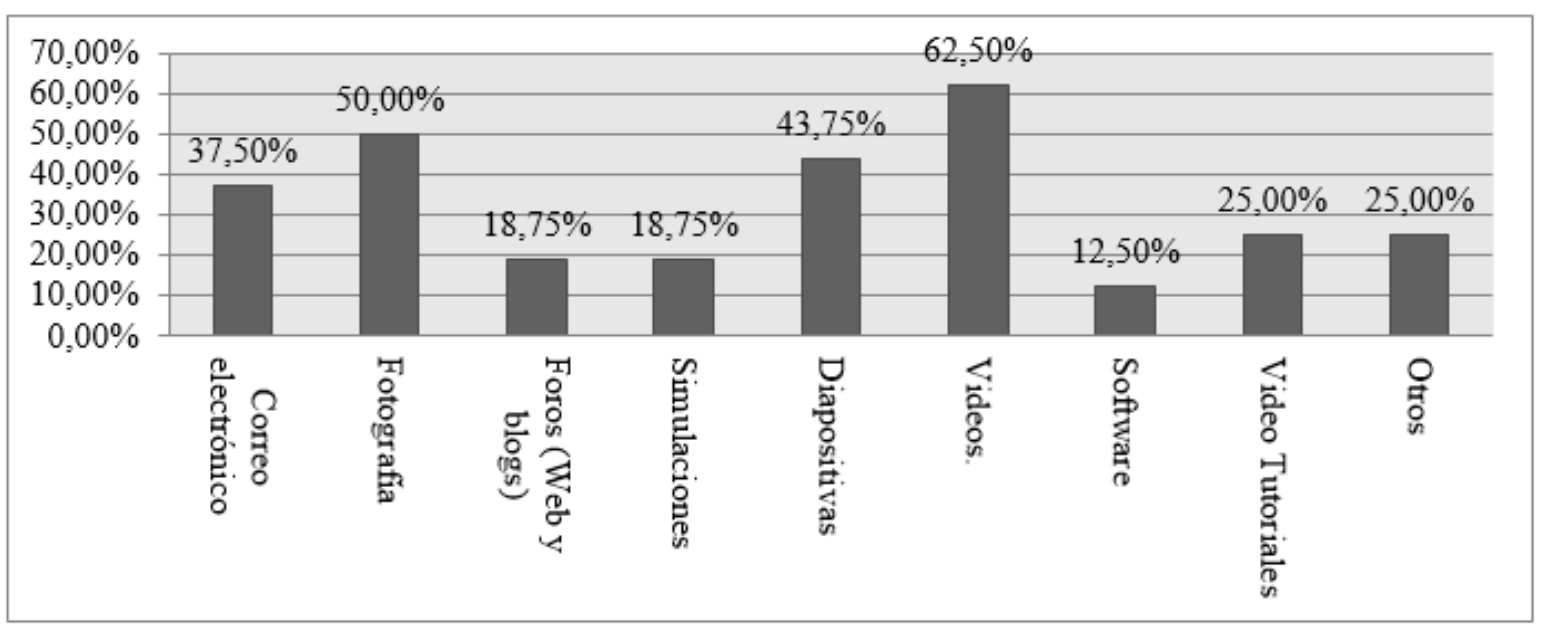

Figura 2. Porcentaje de respuesta a la pregunta: ¿Qué herramientas de las nuevas tecnologías implementa en el desarrollo de su praxis pedagógica?

Fuente: Hernández y Lossada (2018)

El 25\% de los docentes argumentaron que les gustaría implementar otros tipos de herramientas que ofrece las NTIC, tales como apps educativas, web 2.0 y 5.0, plataformas y e-learning".

Para el caso de las encuestas desarrolladas a estudiantes, semejante a la de los docentes en cuanto a alternativas NTIC aplicables se obtienen los siguientes resultados:

- En un $100 \%$ consideran tener ciertas competencias en el manejo y so de las TIC, sin embargo aclaran que no han recibido ningún tipo de formación en estas.

- El 47\% piensa que el uso de las TIC en educación contribuye a desarrollar habilidades de participación y acciones responsables, generando mayor autonomía; un 26\% cree que estas incrementan la creatividad e imaginación y finalmente un $19 \%$ evidencia que contribuyen a desarrollar habilidades de participación y acciones responsables generando mayor autonomía; por el contrario, el $8 \%$ aseguran que estas son una ventajas para los docentes ya que facilitan su labor. 
Revisión documental de las tecnologías de la información y las comunicaciones (TIC) aplicadas en la educación secundaria colombiana, Diego Hernández - Benjamín Losada (57:76)

- Al indagar sobre las herramientas que frecuentemente utilizan sus docentes en el desarrollo de las clases, cabe resaltar que es una pregunta abierta y las opciones de respuesta no son excluyentes, por tanto los estudiantes podían escoger varias opciones para considerar la mayor proporción que las herramientas TIC utilizadas por sus docentes: presentación con diapositivas $88,68 \%$, manejo de software $86,79 \%$, videos $86,79 \%$ y fotografías $58,49 \%$.

- Se puede deducir que los estudiantes experimentan nuevos cambios en la perspectiva educativa cuando sus docentes implementan las TIC en el desarrollo de las prácticas educativas. El $53 \%$ las ve como aliadas para poder afrontar diferentes problemas de su entorno, el 34\% asegura tener mejor comprensión del tema, el 11\% experimenta mayor capacidad de análisis; el $2 \%$ piensa que traen desventajas ya que pueden generar distracción.

La entrevista con los docentes permite reflexionar y argumentar sobre sus vivencias en cuanto al proceso de enseñanza, y como las TIC se involucran en este; aseguran que se debe avanzar hacia un cambio de currículo y del plan educativo institucional, lo que sugiere nuevas prácticas para generar un modelo didáctico basado en las mismas.

Además consideran que su estado actual en Colombia se aleja de las políticas educativas, ya que los recursos en infraestructura rural son escasos con su reflejo en la calidad educativa, ya que el estudiantado tiene pocas posibilidades de interactuar con plataformas que contribuirían a mejorar su rendimiento académico.

Observan que se necesita mayor cualificación de los docentes, ya que un profesor mejor preparado en TIC, genera mayor impacto en la calidad no solo de enseñanza sino en el entorno de aprendizaje de los jóvenes. El MEN debe planear estrategias educativas que conlleven a resignificar el ámbito educativos desde las TIC, mediante mayor infraestructura y conectividad, adecuados equipos, soporte en línea, y aplicaciones.

Sin embargo, señalan que se ha avanzado en estos frentes gracias a organizaciones como "Computadores para Educar" que dota a las instituciones a nivel nacional de computadores, infraestructura, laboratorios tecnológicos y la creación por parte del MEN del portal "Colombia aprende" que genera espacios para el aprendizaje y compartir conocimientos pero debe garantizarse la conectividad ya que sin esta no se puede asignar tareas y acceder al portal y otras plataformas que contribuirían de manera decisiva al proceso de enseñanza-aprendizaje. 
Revisión documental de las tecnologías de la información y las comunicaciones (TIC) aplicadas en la educación secundaria colombiana, Diego Hernández - Benjamín Losada (57:76)

El análisis documental señalado en la fase 3 arrojo los siguientes resultados:

El libro sobre innovación educativa que toma el periodo 2014 y 2016, en criterio de Bedoya et al. (2016) resalta el rezago de conectividad en las zonas rurales del país, es decir, existen políticas educativas de infraestructura que llegan principalmente a zonas urbanas, pero, en áreas rurales es muy bajo el servicio y las dotaciones, produciendo por ende poca interactividad de estudiantes y docentes con recursos tecnológicos y herramientas TIC, generando inequidad entre algunas regiones del país y el sector educativo rural y urbano.

El segundo, la investigación realizada por la Fundación Santillana está relacionado al uso y acceso escolar a las TIC, destacando que los estudiantes que tienen el acceden a las mismas con mayor frecuencia, alcanzan hasta 44 puntos más en las pruebas Saber 11.

Este estudio demostró que los estudiantes que tienen mayor confianza en el uso de las NTIC alcanzan mayores puntajes en las pruebas internas (Supérate, Saber 11) y externas (Pisa) y desarrollaran competencias, destrezas y habilidades más eficaces que aquellos que por determinadas situaciones tienen pocas posibilidades de trabajar con las herramientas de las nuevas tecnologías. No obstante este mismo paradigma se ve reflejado en la educación urbana vs la educación rural, ya que la brecha digital indica que los puntajes tanto en pruebas externas e internas son más sobresalientes en las instituciones ubicadas a nivel urbano.

Se establece que los jóvenes colombianos al terminar secundaria tienen un uso moderado de las TIC, aun cuando en ocasiones el uso del computador lo destinan más hacia el ocio que ha tareas educativas beneficiando otras competencias, como por ejemplo, el aprendizaje autónomo y estrategias meta cognitivas, que a la postre se relacionan directamente con el logro escolar, aun cuando pudiere sonar paradójico.

La investigación en comento sugiere que se deben realizar los esfuerzos posibles para reforzar las TIC en el campo educativo ya que estas generan en el estudiantado destrezas computacionales que contribuyen a un mejor desempeño en las áreas evaluadas, voz a la que se podría unir Francesc (2014) al considerar que la calidad de la educación mejorara sustancialmente si se trabajan 3 elementos: 
Revisión documental de las tecnologías de la información y las comunicaciones (TIC) aplicadas en la educación secundaria colombiana, Diego Hernández - Benjamín Losada (57:76)

- El concepto de la enseñanza como mera transmisión de contenidos debe dejar paso a nuevas tecnologías que posibiliten el desarrollo de las competencias.

- El pilar de la calidad educativa son las competencias profesionales docentes.

- El uso de las tecnologías en educación permite crear entornos de enseñanza y aprendizaje que facilitan el desarrollo de competencias.

Se debe contar con profesorado competente y en condiciones apropiadas, ya que la formación de competencias digitales es determinante en el ámbito educativo como pre requisito además para su inclusión en la llamada sociedad del conocimiento. (Francesc, 2014). 
Revisión documental de las tecnologías de la información y las comunicaciones (TIC) aplicadas en la educación secundaria colombiana, Diego Hernández - Benjamín Losada (57:76)

\section{CONCLUSIONES}

El estudio de caso realizado y los análisis realizados a través de la documentación y antecedentes investigativos, denotan una clara preocupación por la formación docente; si bien es cierto que el gobierno nacional en los últimos años ha invertido en cursos de capacitación con programas como SoyTIC, Innovatic, TecnoTIC, EduTIC entre otros, se deben generar esfuerzos para tener docentes mejor preparados en el campo de las TIC para mejorar las competencias de los estudiantes y condiciones para alcanzar logros conjuntos, lo que dará como resultado una mejor calidad educativa.

Otro aspecto es la creación de un modelo didáctico a partir de las TIC: si se planea dicho modelo estratégicamente en el campo educativo, será más fácil guiar a los docentes y estudiantes a la consecución de las metas académicas y un mejor desempeño profesional y académico; el realizar un nuevo modelo implica cambio de paradigma desde la óptica docente reflejada en el "Informe Nacional de Competitividad de 2017", con la creación de un modelo didáctico que genere a partir de las TIC mejores resultados en el proceso de enseñanza-aprendizaje en Colombia.

Este nuevo modelo, apunta a generar nuevos aprendizajes, cambio curricular, definición de herramientas TIC según las áreas del conocimiento y de aplicación, capacitación docente de alta calidad, nuevos logros y roll docente.

El gobierno nacional por medio del MEN debe crear nuevas políticas educativas que permitan garantizar la óptima competitividad y la adquisición de nuevos estándares en el desarrollo e impulso de mecanismos que contribuyan a garantizar la calidad de la educación mediante las NTIC, que permitan estar a la altura de la educación mundial.

Los docentes muestran gran interés para involucrar las TIC en su praxis pedagógica; entre las herramientas que destacan están las webs desde la 2.0 a las 5.0, simulaciones, videotutoriales, apps educativas y la optimización de la conectividad. 
Revisión documental de las tecnologías de la información y las comunicaciones (TIC) aplicadas en la educación secundaria colombiana, Diego Hernández - Benjamín Losada (57:76)

\section{REFERENCIAS BIBLIOGRÁFICAS}

Bakia, M., Murphy, R., Anderson, K \& Trinidad, G (2011). International Experiences with Technology inEducation: Final Report. Washington, D.C..U.S. Department of Education.

Bedoya, F., Hernández, L., Rivera, P \& Ferro, M., (2016). La Innovación Educativa en Colombia. Bogotá. Comité Editorial: CIEN, Ministerio de Educación Nacional - Universidad Nacional.

Cabero, J. (2000). Nuevas tecnologías aplicadas a la educación. Editorial Síntesis. España.

Castells, M. (1998). La era de la información. Economía, sociedad y cultura. La Sociedad Red. Vol ( $\left.\mathrm{n}^{\circ} 1\right)$. Alianza Editorial.

Castellar, E. (2011). Diagnóstico del uso de las TIC en estudiantes de colegios oficiales del municipio de Soledad (Atlántico). Revista Zona próxima. No. 14. (Pp. 74-89).

Cardenas, A. (2015). Implicaciones de las TIC en la formación profesional del docente en la educación básica y media. Recuperado de: http://recursos.portaleducoas.org/sites/default/ files/5107.pdf

Consejo Privado de Competitividad. (2017). Informe Nacional de Competitividad 2016-2017. Recuperado de: https://compite.com.co/informe/informe-nacional-decompetitividad-2016-2017/.

Francesc, P. (2014). Tecnologías para la mejora de la educación: experiencias de éxito y expectativas para el futuro. Fundación Santillana. Recuperado de:https://www.mecd.gob.es/ dam/jcr...155b.../docbasico2015semeducsantillana-pdf.pdf.

Hermosa, P. (2015). Influencia de las tecnologías de información y comunicación (TIC) en el proceso enseñanza-aprendizaje: una mejora de las competencias digitales. Revista científica José María Córdoba. Vol 13 Nº16, pp. 121-132. 
Revisión documental de las tecnologías de la información y las comunicaciones (TIC) aplicadas en la educación secundaria colombiana, Diego Hernández - Benjamín Losada (57:76)

Hernández, D \& Martínez, J. (2014). Aplicación de herramientas Web 2.0 para simulación de situaciones de optimización matemática en la Institución Educativa San Marcos de Muzo. (Tesis inédita de maestría). Universidad de Santander. Colombia.

Latorre, A. (2003). La Investigación Acción. Conocer y cambiar la practica educativa. Editorial Grao. España.

Meneses, G. (2007). NTIC, interacción y aprendizaje en la universidad. (Tesis de doctorado, Universitat Rovira I Virgili). Recuperada de https://www.tesisenred.net/bitstream/ handle/10803/8929/2Lasnuevastecnologiasdelainformacion.pdf? sequence $=8$.

Muñoz, J. (2012). Apropiación, uso y aplicación de las TIC en los procesos pedagógicos que dirigen los docentes de la Institución Educativa Núcleo Escolar de Rural Corinto. Maestría en enseñanza de las ciencias exactas y naturales. Universidad Nacional de Colombia. Colombia.

Parra, C. (2011). TIC, conocimiento, educación y competencias en la formación de maestros. No 36, pp 145-159. Revista Nomadas. Colombia. Recuperado de: http://www.redalyc.org/ pdf/1051/105124264010.pdf

Tique, E. (2010). Estado Actual de la Aplicación de las TIC en los Colegios que imparten el Ciclo de Educación Media de las Zonas Rurales del Municipios de Ibagué. Maestría en educación. Universidad del Tolima. Colombia.

Ulate, M. (2012). Las TIC en educación primaria y secundaria: estado del arte. Revista Diálogos. Año 7, $\mathrm{N}^{\circ} 11$, pp. 7-27.

Visbal,H. (2018). Las TIC y la educación, un reto para el aprendizaje. Las 2 orillas. Recuperado de: https://www.las2orillas.co/las-tic-y-la-educación-un-reto-para-el-aprendizaje/. 